\title{
Uso do QFD para definir requisitos necessários para a aplicação da estratégia da modularidade nos fornecedores de primeiro nível do setor automotivo
}

\section{Use of QFD to define requisites necessaries for the application of the modularity strategy in the first level suppliers of the automotive sector}

José Antonio Carnevalli ${ }^{1}$

José Eduardo Rodrigues de Sousa ${ }^{2}$

Samuel Carvalho de Benedicto ${ }^{3}$

Charles Gauguin Alfaia Medeiros ${ }^{4}$

Marcos Ricardo Rosa Georges ${ }^{5}$

1 Pós-Doutorado - Universidade de São Paulo e Doutorado em Programa de Pós Graduação Engenharia de Produção - Universidade Metodista de Piracicaba. PUC-Campinas jose.carnevalli@puc-campinas.edu.br

2 PUC-Campinas eduardo.sousa@puc-campinas.edu.br

3 PUC- Campinas samuel.benedicto@puc-campinas.edu.br

4 PUC-Campinas charles_gauguin@hotmail.com

5 PUC-Campinas marcos.georges@puc-campinas.edu.br

\section{Resumo}

Este trabalho tem como objetivo realizar um estudo da aplicação teórica do QFD, para definir requisitos necessários à aplicação da estratégia modular nos fornecedores de primeiro nível das montadoras do setor automotivo. Este projeto envolveu a construção da Matriz da Qualidade teórica utilizando dados da literatura para identificar os requisitos necessários a aplicação da modularidade. Com a conclusão da matriz da qualidade, verificou-se que os aspectos de infraestrutura estão relacionados com: (i) treinamento, experiência na função e nível de qualificação; (ii) gestão da qualidade; (iii) a busca da excelência e do know how, e; (iv) ter eficiência na logística. Somente após desenvolver estas competências em qualidade, logística e Know how a empresa estará pronta para assumir como fornecedora de módulos numa relação de parceria estratégica com a montadora.

Palavras chave: QFD. Modularidade. Terceirização. Setor automotivo.

\section{Abstract}

This work is aimed to conduct a study of the theoretical application of QFD (Quality Function Deployment) to define requisites necessaries for the application of the modular strategy in the first level suppliers of the car assembly plants. This project involved the construction of the theoretical Quality Matrix utilizing literature data to identify the requisites necessaries for the application of modularity. With the conclusion of the Quality Matrix, it was found that the aspects are related with: (i) training, experience in the function and qualification level; (ii) quality management; (iii) the search for excellence and know how; (iv) having efficiency in the logistics. Only after developing these competencies in quality, logistics and Know how, the enterprise will be ready to take over as a supplier of modulus in a relationship of strategic partnership with the car assembly plant.

Keywords: QFD. Modularity. Outsourcing. Automotive sector. 


\section{Introdução}

A aplicação da estratégia modular surgiu antes da segunda guerra mundial sendo aplicada na indústria de construção naval. Entretanto, conforme relatado por Elphick (2006) o uso em grande escala desta estratégia foi primeiramente aplicada na construção dos navios de transporte de tropas da classe Liberty na década de 1940 nos EUA. Inicialmente, limitada como estratégia de subconjuntos pré-montados, para agilizar a fabricação naval, a modularidade tem o seu aprimoramento na década de 1960 na IBM, como pode ser visto no texto de Baldwin e Clark (2000) em relação à indústria de informática. Já na década de 1990 o setor automobilístico também passou a aplicar a estratégia modular aumentando a desverticalização do setor, com a criação de parcerias estratégicas entre montadoras e fornecedores, como se verifica no trabalho de Doran (2003).

Ainda neste setor surgiram três tipos de organização de plantas produtivas das montadoras, as quais são:

- Condomínio Industrial onde os fornecedores de $1^{\text {a }}$ camada estão instalados dentro do terreno da montadora e entregam seus módulos na linha de produção da montadora;

- Consórcio Modular: os fornecedores de $1^{\mathrm{a}}$ camada estão instalados dentro da fábrica da montadora e são responsáveis pela linha de montagem do veículo;

- Parques industriais: os fornecedores de $1^{\mathrm{a}}$ camada estão instalados em até um quilômetro da planta da montadora, em terrenos próprios do fornecedor. Estes entregam seus módulos na montadora (Pires \& Sacomano, 2010; Pires 2009; Larsson, 2002).

Como principal benefício desta estratégia, a modularidade facilita a desverticalização das montadoras, pois os produtos são divididos em grandes subconjuntos (módulos) específicos, permitindo que a fabricação e montagem possam ser realizadas separadamente em diferentes lugares - Inclusive internacionalmente (Segismundo \& Miguel Cauchick, 2014; Veloso \& Fixson, 2001), ou seja, facilita a terceirização (Morris \& Donnelly, 2006).

Entretanto, a literatura tem identificado várias dificuldades na aplicação da estratégia modular no setor automobilístico. As principais são:

- Dificuldades de encontrar fornecedores capacitados, o que pode limitar o projeto dos módulos ao know how dos fornecedores disponíveis (Carnevalli, Miguel \& Salerno, 2013);

- Dificuldade de estar capacitado para aplicar a modularidade (Carnevalli et al., 2013; Prieto \& Miguel, 2011; Rodrigues, Carnevalli \& Miguel, 2012; Miguel, 2009; Pandremenos, Paralikas, Salonitis \& Chryssolouriset, 2009; Salerno, Marx, Zilbovicius \& Dias, 2009). Dentre elas podem-se citar:

- Dificuldade de projetar produtos modulares: - Necessidade de adaptação no projeto do produto (Rodrigues et al., 2012);

- A necessidade de reprojetar o produto e o processo (Hoek \& Weken, 1998);

- A definição de métodos apropriados para subdividir um produto em módulos (Asan, Polat \& Serdar, 2004; Gadde \& Jellbo, 2002);

- Estar capacitado para fabricar os módulos (Carnevalli et al., 2013; Prieto \& Miguel, 2011; Rodrigues et al., 2012; Miguel, 2009; Pandremenos et al., 2009; Salerno et al., 2009);

- Aumento da verticalização entre fornecedores para ter mais controle na produção dos módulos na sua própria linha e facilitar o de- 
senvolvimento dos módulos para topicalização e padronização do produto (Carnevalli et al., 2013; Prieto \& Miguel, 2011; Rodrigues et al., 2012; Miguel, 2009; Pandremenos et al., 2009; Salerno et al., 2009);

- Aumento da verticalização em P\&D e do investimento no fornecedor (Carnevalli et al., 2013; Prieto \& Miguel, 2011; Rodrigues et al., 2012; Miguel, 2009; Pandremenos et al., 2009; Salerno et al., 2009);

- Atingir o nível de maturidade em modularidade para permanecer competitivo num contexto de parcerias globais (Carnevalli et al., 2013; Prieto \& Miguel, 2011; Rodrigues et al., 2012; Miguel, 2009; Pandremenos et al., 2009; Salerno et al., 2009);

- A necessidade de investimentos do fornecedor para estar capacitado para fornecer os módulos (Arnheiter \& Harren, 2005; Doran, 2002; Pires, 1998; Collins, Bechler \& Pires, 1997);

- Dificuldades de capacidade produtiva e logísticas tais como:

- Dificuldades dos fornecedores em ter operações flexíveis à demanda (Carnevalli et al., 2013; Prieto \& Miguel, 2011; Rodrigues et al., 2012; Miguel, 2009; Pandremenos et al., 2009; Salerno et al., 2009);

- Dificuldade de ter que entregar os módulos com frequência diária na montadora (Salerno et al., 2009);

- Entregar pontualmente os módulos diretamente na linha de produção da montadora (Carnevalli et al., 2013; Prieto \& Miguel, 2011; Rodrigues et al., 2012; Miguel, 2009; Pandremenos et al., 2009; Salerno et al., 2009);

o Investimento do fornecedor na criação de linhas de montagem de módulo na planta da montadora (Prieto \& Miguel, 2011);
- Dificuldade de realizar a integração das capacidades dos vários fornecedores de diferentes módulos (Pandremenos et al., 2009);

- Falta de alinhamento entre os setores da empresa durante o desenvolvimento do projeto do produto e do processo de produção, principalmente com o setor de compras que tem papel importante nas etapas de seleção de fornecedores (Miguel, 2009);

- Aumentar a dependência com as montadoras (Carnevalli et al., 2013; Prieto \& Miguel, 2011; Rodrigues et al., 2012; Miguel, 2009; Pandremenos et al., 2009; Salerno et al., 2009; Frigant \& Lung, 2002).

Deste modo, verifica-se que o uso da modularidade gera várias dificuldades para o fornecedor de módulo, relacionadas a estar capacitado para aplicar a estratégia na fase de projeto, fabricação e expedição. Por isto, a importância de estudar este tema que é cada vez mais aplicado no setor automobilístico.

O objetivo deste trabalho é realizar um estudo da aplicação teórica do QFD, para definir requisitos necessários à aplicação da estratégia modular nos fornecedores de primeiro nível das montadoras do setor automotivo. Para tanto, foi realizada uma revisão de literatura sobre o tema e com os dados desta revisão foi feita a aplicação teórica do QFD. De modo específico, a pesquisa possui os seguintes objetivos:

- Identificar os recursos e competências necessários para a aplicação da modularidade;

- Identificar as alterações de relacionamento entre a empresa (fornecedor de $1^{\circ}$ nível) com seus fornecedores (fornecedor de $2^{\circ}$ nível) que são necessárias para se capacitar na aplicação da modularidade;

- Identificar os critérios de responsabilidade pela qualidade dos módulos. 
A seguir serão apresentados os métodos de pesquisa utilizados.

\section{Uso do método do QFD}

Criado na década de 1960 para garantir a qualidade do produto desde a fase de projeto (Akao, 1996), o método do QFD é formado por três unidades básicas (Cheng \& Melo Filho, 2007):

- As tabelas onde os dados são organizados utilizando o método KJ (diagramas de afinidades e diagrama em árvore);

- As matrizes que são a união de duas tabelas, sendo uma desdobrada pelos elementos da outra, como no caso da primeira matriz (matriz da qualidade) onde os requisitos dos clientes (QE - qualidade exigida) são desdobrados na tabela de características da qualidade (CQ) que são as especificações de projeto mensuráveis para atender os requisitos dos clientes. Nestas matrizes são definidas as prioridades do projeto.

- Modelo Conceitual: são os demais desdobramentos (tabelas e matrizes) necessários para se atingir os objetivos de desempenho definidos na primeira matriz. Este modelo pode ou não ser necessário conforme os objetivos da aplicação do QFD.

Mais recentemente o método do QFD vem sendo utilizado em várias aplicações como desenvolvendo um método para projetar produtos plataformas, (Park, Shin, Insun \& Hyemi, 2008); desenvolvimento de produtos e seus módulos (Wang \& Chen; 2012); desenvolver um novo modelo de desenvolvimento de produtos sustentáveis (Salari \& Bhuiyan, 2016); Desenvolvimento ambiental sustentável com o ANP e o QFD (Lam \& Lai, 2015) e Desenvolvimento de uma estrutura para criação de produtos sustentáveis usando o QFD e a lógica fuzzy (Younesi \& Roghanian, 2015). Também é utilizado para a implantação do sistema de gestão da qualidade (Brad, 2008); aplicar o lean production (Zarei, Fakhrzad \& Paghaleh, 2011); desenvolver um novo currículo de graduação em Gestão da Cadeia de Suprimentos (Gonzalez, Quesada, Gourdin \& Hartley, 2008); projetar serviços logísticos modulares (Yond \& Saara, 2011); para definir a localização das fábricas dos módulos que formam o produto (Grauer, 2007); formular estratégias em organizações de ensino (Zohbabi \& Manteghi, 2011); desenvolver estratégias de produção (Jia \& Bai, 2011); para o planejamento de estratégia (Killen, Walker \& Hunt, 2005); desenvolver um método de tomada de decisão em investimentos na construção naval (Celik, Cebi, Kahraman \& Er, 2009); desenvolver uma estratégia para a fábrica (Pur \& Tabriz, 2012); fazer o alinhamento entre estratégia competitiva com estratégia da cadeia de suprimentos (Katta, Kambagowni \& Kandukuri, 2012) e criar um método de seleção de fornecedores (Ni \& Deng, 2007; Ho, Dey \& Lockström, 2011; Bhattacharya, Geraghty \& Young, 2010; Ho, He, Lee \& Emrouzneja, 2012; Sorroor, Tarokh \& Abedzadeh, 2012).

Nesta pesquisa resolveu-se utilizar o método do QFD para definir requisitos necessários à aplicação da estratégia modular. O método consiste na elaboração de uma ou mais matrizes, de modo que estes requisitos possam ser atendidos, através de vários desdobramentos, quando necessário.

\section{Método de pesquisa e plano de análise dos dados}

Neste itens serão apresentados os métodos de pesquisa utilizados neste estudo. 


\subsection{Pesquisa bibliográfica}

Para a pesquisa bibliográfica foram utilizadas as quatro etapas sugeridas por Marconi e Lakatos (2002): Identificação, Localização, Obtenção e Cadastramento das Fontes Bibliográficas.

Para a identificação e localização dos textos de interesse no caso do QFD utilizou-se consulta a base de dados do Portal de Periódicos da CAPES (Coordenação de Aperfeiçoamento de Pessoal de Nivel Superior) onde se encontram os artigos internacionais, através da busca por assunto específico. As palavras chave foram definidas buscando artigos que tenham informações relevantes para atender aos objetivos da pesquisa, os quais são:

- Aplicações do QFD para implementar a modularidade através das palavras chave Modular e QFD;

- Aplicações do QFD para implementar estratégias de Gestão da Cadeia de Suprimentos através das palavras chave SCM e QFD;

- Aplicações do QFD para implementar Outsourcing através das palavras chave Outsourcing e QFD;

- Estudar Terceirização e critérios de seleção de fornecedores no setor automobilístico através das palavras chave Outsourcing, Auto, Automotive e Automobile;

- Também foi realizada uma revisão dos dados brutos dos fichamentos sobre modularidade no setor automobilístico realizado pelos proponentes num estudo anterior. Nesta pesquisa anterior foram utilizadas as seguintes palavras chave modular: Modularization e Modularity.

\subsection{Aplicação do QFD}

Para aplicar o QFD foi utilizada uma adaptação do roteiro desenvolvido por Carnevalli et al. (2010), sendo que algumas etapas não foram realizadas por ser pesquisa teórica. Segue resumidamente a sequência aplicada após a adaptação:

1 Utilização dos dados da literatura dos fichamentos para preencher a tabela de qualidade exigida aplicando os diagramas de afinidades e de árvore (também conhecido como método $\mathrm{Kj}$ ) para organizar e detalhar os requisitos dos clientes analisados;

Para definir o grau de importância da QE (Qualidade exigida) utilizou-se o questionário de Kano conforme os procedimentos desenvolvidos por Tontini (2003, 2007). Sendo está uma pesquisa teórica, esta aplicação do questionário de Kano foi realizada pelos proponentes. Este questionário utiliza uma escala que visa avaliar a satisfação ou não pela existência ou ausência de um requisito. Se a presença do requisito ocorrer ele será avaliado como 1- Gosta; 2 - Óbvio; 3 - Neutro; 4 Indiferente; 5- Não gosta. A mesma escala é utilizada quando o requisito está ausente. Ao comparar a duas respostas, Tontini (2003) consegue classificar a satisfação dos clientes como: (A) Atrativo (requisito não previsto pelo cliente, mas cuja presença supera a expectativa de forma positiva); (O) Linear (conforme aumenta o número de requisitos atendidos mais satisfeito fica o cliente); (M) Óbvia (a presença do requisito não gera satisfação, mas a sua ausência causa insatisfação por ser considerado um requisito padrão do produto); (I) Indiferente (a presença ou a ausência ao requisito não causa nenhuma reação nos clientes); (R) Reverso, ou; (Q) Questionável. Esta classificação é definida através da combinação das respostas das duas escalas conforme apresentados a seguir (Tontini, 2003):

- A: Qualidade Atrativo: é a combinação da resposta 1-gostar por existir o requisito com 
uma das respostas 2 - Óbvio; 3 - Neutro; 4Indiferente por não ter os requisitos;

- O: Qualidade Linear é a combinação das resposta 1-gostar por existir o requisito com a resposta 5 - Não gosta por não ter o requisito;

- M: Qualidade Óbvia é a combinação de uma das respostas 2- Óbvio; 3- Neutro; 4Indiferente por existir o requisito com a resposta 5 - Não gosta por não ter o requisito;

- I: Indiferente é a combinação de uma das respostas 2- Óbvio; 3 - Neutro; 4- Indiferente por existir o requisito com uma das respostas 2- Óbvio; 3- Neutro; 4- Indiferente por não ter o requisito;

- R: Reverso é a combinação de uma das respostas 2- Óbvio; 3- Neutro; 4-Indiferente por existir o requisito com a resposta 1- gosta por não ter o requisito;

- Q: Questionável é a combinação das respostas 5-Não gostar por existir o requisito com a resposta 5- Não gosta por não ter o requisito.

2 Após a classificação de cada QE em A, O, M, I, foi aplicada a seguinte fórmula desenvolvida por Tontini (2003, 2007): (1 + MAX(ISI| $|\mathrm{DI}|)$ onde $\% \mathrm{SI}=(\mathrm{A}+\mathrm{O}) /(\mathrm{A}+\mathrm{O}+\mathrm{M}+\mathrm{I})$ (porcentagem de satisfação) e \%DI $=(\mathrm{M}+\mathrm{O}) /$ $((\mathrm{A}+\mathrm{O}+\mathrm{M}+\mathrm{I}) \times(-1))$ (porcentagem de insatisfação) para calcular os pesos da QE.

3 Priorizou as QEs que farão parte da matriz do QFD, buscando reduzir o tamanho da matriz, fazendo uma análise crítica dos itens das QEs. Para isto os requisitos da qualidade classificados como I: Indiferente e R: Reverso segundo o modelo de Kano proposto por Tontini $(2003,2007)$ não foram considerados.

4 Realizou o desdobramento das QEs em CQs (Características da Qualidade);
5 Realizou as correlações entre os QEs com os CQs, pela seguinte sequência: Iniciou as correlações com as QEs que geram cada CQs, para depois verificar a existência de correlações entre as QEs com as CQs que elas não geraram;

6 Os itens prioritários das CQs foram definidos através da conversão do peso relativo das QEs pelas correlações com CQs.

\section{Análise e discussão dos resultados}

Nesta etapa da pesquisa foram fichados 28 artigos, uma tese, uma dissertação e uma norma (ABNT ISO/TS 16949:2010), sendo 17 sobre o QFD. Também Foi realizada uma revisão dos dados brutos dos fichamentos sobre modularidade no setor automobilístico realizado pelos proponentes num estudo anterior.

Os resultados das tabelas Qualidade Exigida; Características da Qualidade e da Matriz de Qualidade são apresentados no tópico 4.1, 4.2 e 4.3 a seguir.

\subsection{Resultado da matriz da qualidade em relação da tabela de qualidades exigidas}

$\mathrm{Na}$ aplicação teórica do QFD foi realizada a matriz da qualidade formada pelas tabelas Qualidades Exigidas (QEs) e Características da Qualidade (CQs). Em relação às Qualidades Exigidas identificadas com os dados de análise de literatura, verificou-se a formação de nove agrupamentos de requisitos dos clientes (ver tabela 1). Estes estão relacionados com:

- A maturidade da empresa, no sentido de não ser iniciante no segmento que atua, de modo 
a já ter o nível de infraestrutura, qualidade e Know-how necessários;

- A reputação positiva da empresa na visão dos seus clientes, relacionada à qualidade dos serviços e produtos oferecidos;

- Atender as exigências de qualidade dos produtos e serviços oferecidos, ou seja, não basta ter uma boa reputação, é necessário que ela seja verdadeira;

- Desenvolver a área de logística, por exemplo, aspectos de entregas como rapidez de atendimento dos pedidos e flexibilidade;

- Ser competitivo em relação aos custos quando comparados com os concorrentes;

- Know-How no segmento, relacionado com as competências necessárias para projetar, fabricar e entregar os módulos conforme as exigências dos clientes;

- Aspectos financeiros, como por exemplo, baixos riscos de comprometimento das receitas (Borghi, 2011), o que pode ser verificado analisando a existência ou não da lucratividade e ter recursos internos para investir;

- Adequado relacionamento com os clientes. Este aspecto é muito importante para um fornecedor de módulos para a montadoras, pois é necessário criar uma parceria entre as empresas, visto que informações de programação da produção tem que ser compartilhas entre as duas empresas. Isto ocorre porque em muitos casos os módulos devem ser entregues de forma sincronizada na linha de montagem da montadora;

- Riscos associados a empresa, no sentido de que o cliente saiba qual os índices de risco de ocorrer falhas no produto e ou serviço fornecido e do fornecedor ter problemas financeiros.

Verificou-se que dos 52 requisitos da qualidade identificados, três se destacam com maior grau de importância na matriz do QFD, os quais são:

Tabela 1: Apresentação de parte da tabela Qualidade Exigida (Principais resultados)

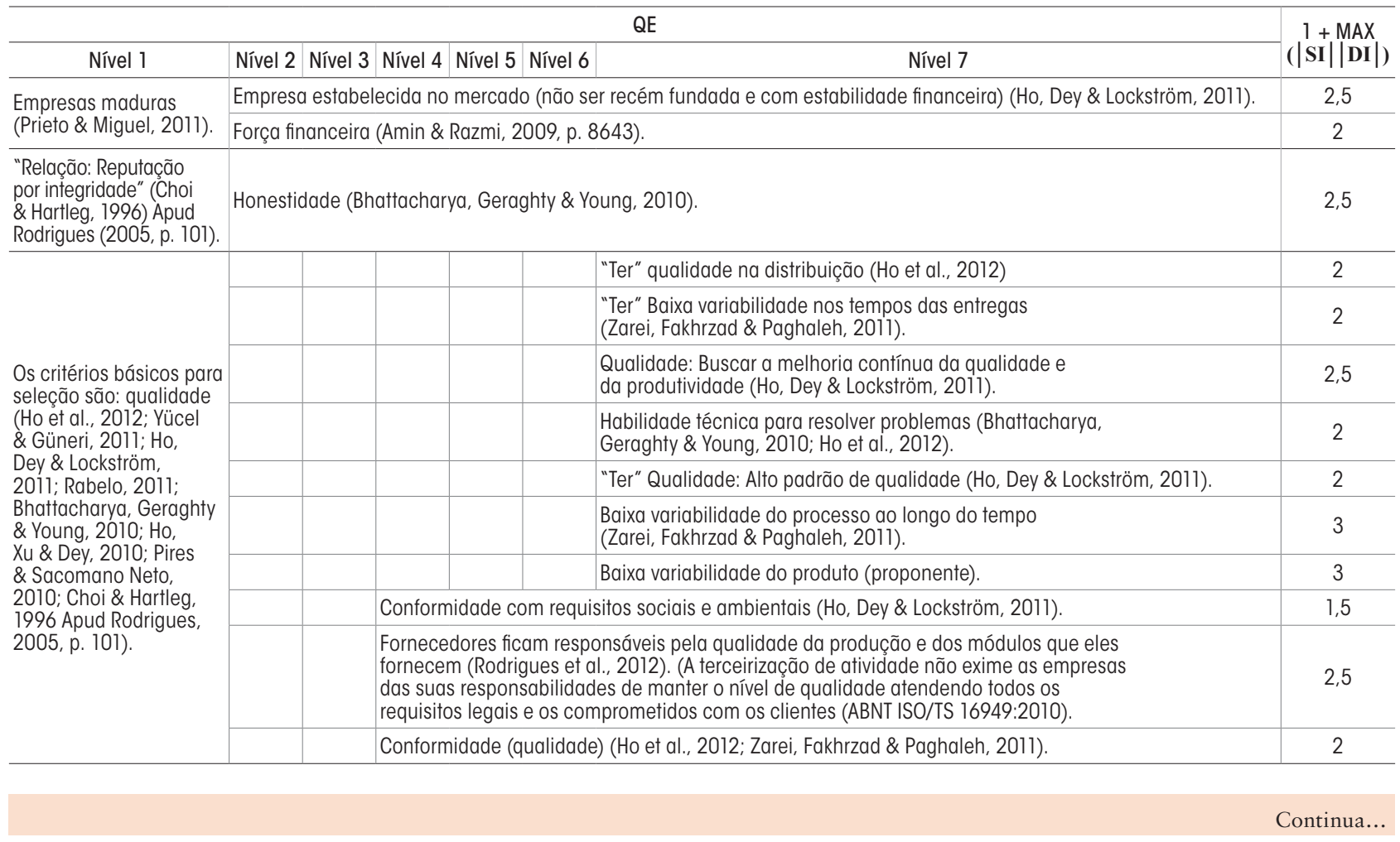




\section{Continuação Tabela 1: Apresentação de parte da tabela Qualidade Exigida (Principais resultados)}

\begin{tabular}{|c|c|c|c|c|c|c|}
\hline \multicolumn{6}{|r|}{ QE } & \multirow{2}{*}{$\begin{aligned} 1+\operatorname{MAX} \\
(|\mathbf{S I}||\mathbf{D I}|)\end{aligned}$} \\
\hline Nível 1 & Nível 2 & Nível 3 & Nível 4 & Nível 5 & Nível 6 & \\
\hline \multirow{12}{*}{$\begin{array}{l}\text { Avaliação de } \\
\text { fornecedores atuais: } \\
\text { avaliar o desempenho } \\
\text { da área de logística } \\
\text { (Pires, \& Sacomano } \\
\text { Neto, 2010). }\end{array}$} & & & & \multicolumn{2}{|c|}{$\begin{array}{l}\text { "Consistência:Resposta rápida" (Katta, Kambagowni \& Kandukuri, } \\
\text { 2012; Choi \& Hartleg, 1996) apud Rodrigues (2005, p. 101). }\end{array}$} & 2 \\
\hline & & & & & $\begin{array}{l}\text { Trabalhar com o mínimo de estoque (Zarei, Fakhrzad } \\
\text { \& Paghaleh, 2011; Jis \& Pires, 2002). }\end{array}$ & 2 \\
\hline & & & & & Kaizen (Pires, 2002). & 2 \\
\hline & & & & \multicolumn{2}{|r|}{ Consistência de entrega (Choi \& Hartleg, 1996) apud Rodrigues (2005, p. 101). } & 2 \\
\hline & & & & & Rapidez nas entregas (Zarei, Fakhrzad, \& Paghaleh, 2011). & 2 \\
\hline & & & & \multicolumn{2}{|c|}{ Precisão (Sorroor, Tarokh \& Abedzadeh, 2012). } & 2 \\
\hline & & & & & $\begin{array}{l}\text { Indicadores logísticos: "flexibilidade no atendimento" } \\
\text { (Pires \& Sacomano Neto, 2010, p. 181). }\end{array}$ & 2 \\
\hline & & & & & Administração: Flexibilidade na adaptação para mudança. (Ho, Dey \& Lockström, 2011). & 2 \\
\hline & & & & & "Ter operações flexíveis à demanda" (Carnevalli et al., 2013, p. 335). & 2,5 \\
\hline & & & & & Entregas: Condições de entregas (Ho et al., 2012). & 2 \\
\hline & \multicolumn{5}{|c|}{ Avaliação de fornecedores ałuais: Logística: Ter inovações em logística (Pires \& Sacomano Neto, 2010). } & 2 \\
\hline & \multicolumn{5}{|c|}{ Desenvolver habilidade de entregar módulos na linha de produção da montadora (Carnevalli et al., 2013). } & 2 \\
\hline \multirow{6}{*}{ Custos (Ho et al., 2012). } & \multicolumn{5}{|c|}{ Avaliar custos (Pires \& Sacomano Neto, 2010). } & 2 \\
\hline & \multicolumn{5}{|c|}{ Custos: Ser proativo na redução de custos (Ho et al., 2012; Ho, Dey \& Lockström, 2011). } & 1,5 \\
\hline & \multicolumn{5}{|c|}{ Custos: Responsabilidade para garantir redução de custos (Ho et al., 2012; Ho, Dey \& Lockström, 2011). } & 1,5 \\
\hline & & & \multicolumn{3}{|c|}{ Custos: Transparências nas questões de custo (Ho, Dey \& Lockström, 2011). } & 2 \\
\hline & & & \multicolumn{3}{|c|}{ Reduzir os custos logísticos totais (Ho et al., 2012). } & 2 \\
\hline & \multicolumn{5}{|c|}{ Preço competitivo (Choi \& Hartleg, 1996) apud Rodrigues (2005, p. 101). } & 2,5 \\
\hline \multirow{10}{*}{$\begin{array}{l}\text { Escolha de fornecedores } \\
\text { para o Condomínio } \\
\text { Industrial devido o seu } \\
\text { Know-How (Pires \& } \\
\text { Sacomano Neto, 2010). }\end{array}$} & & & \multicolumn{3}{|c|}{ Fazer o projeto de fabricação (Zarei, Fakhrzad \& Paghaleh, 2011). } & 2 \\
\hline & & & \multicolumn{3}{|c|}{ Tecnologia: Técnica/ habilidade de inovação (Ho, Dey \& Lockström, 2011). } & 2 \\
\hline & & & Ter admi & inistraçã & io de conhecimento (Zarei, Fakhrzad \& Paghaleh, 2011). & 2 \\
\hline & & & & $\begin{array}{l}\text { Capacid } \\
\text { Hartleg, }\end{array}$ & $\begin{array}{l}\text { dade Tecnológica: Capacidade de projeto (Choi \& } \\
\text { 1996) Apud Rodrigues (2005, p. 101). }\end{array}$ & 2 \\
\hline & & & & Capacid & dade de desenvolver produtos modulares em co-design (Prieto \& Miguel, 2011). & 2 \\
\hline & & & & & $\begin{array}{l}\text { Capabilidade técnica (Bhattacharya, Geraghty \& Young, 2010). } \\
\text { (Choi \& Hartleg, 1996) apud Rodrigues (2005, p. 101). }\end{array}$ & 2 \\
\hline & & & & & Ter competência de fabricação dos módulos (Rodrigues, 2005). & 3 \\
\hline & & & Principai & is critério & os: capacidade de fabricação (Ho, Xu \& Dey, 2010). & 2 \\
\hline & & & $\begin{array}{l}\text { Setor de } \\
\text { durante }\end{array}$ & $\begin{array}{l}\text { compra } \\
\text { o planejc }\end{array}$ & $\begin{array}{l}\text { Is definir requisitos de seleção de fornecedores } \\
\text { amento do produto (proponente). }\end{array}$ & 2 \\
\hline & Ter expe & eriência $n$ & no mercac & do intern & רacional (Prieto \& Cauchick, 2011). & 2 \\
\hline & & & Financei & iro: regra & as de desempenho (Choi \& Hartleg, 1996) apud Rodrigues (2005, p. 101). & 2 \\
\hline & & & Financei & iro: trans & sparência dos dados financeiros (Choi \& Hartleg, 1996) apud Rodrigues (2005, p. 101). & 2 \\
\hline \& Dey, 2010). & & & & & Ter recursos internos para investir (proponente). & 2 \\
\hline & & & & & Baixos riscos de comprometimento das receitas (Borghi, 2011). & 2 \\
\hline & & Critérios & s qualitati & ivos: Alia & ança estratégica (Amin \& Razmi, 2009, p. 8643). & 2 \\
\hline Principais critérios: & & Relacion & namento: & Transpa & arência de longo prazo da Estratégia (Ho, Dey \& Lockström, 2011). & 2,5 \\
\hline Xu \& Dey, 2010). & & & Ter Acess & sibilidad & e (Amin \& Razmi, 2009). & 2 \\
\hline & & & Transferê & ência de & P\&D da matriz para a filial (Borghi, 2011). & 2 \\
\hline & & Riscos d & de falta de & e habilid & ade de identificar e prevenir potenciais problemas (Ho et al., 2012). & 2 \\
\hline $\begin{array}{l}\text { Riscos (Ho, Xu \& Dey, } \\
\text { 2010: Ho et al. 2012). }\end{array}$ & & Riscos $d$ & de falta de & e acessib & bilidade e facilidade de conectar-se com pessoas nas emergências (Ho et al., 2012). & 2 \\
\hline & & & & & Baixos riscos de recursos externos (proponente). & 2 \\
\hline
\end{tabular}

Fonte: Elaborada pelos autores.

- Baixa variabilidade do processo ao longo do tempo (Zarei, Fakhrzad \& Paghaleh, 2011);

- Baixa variabilidade do produto (proponente);
- Método de seleção dos fornecedores pela competência de fabricação do módulo (Rodrigues, 2005). 
Deste modo, os pontos mais importantes na seleção de fornecedores modulares são empresas que conseguem manter um nível de qualidade constante e tenham o conhecimento necessário para a fabricação dos módulos. Além destes, outros setes requisitos de qualidade se destacaram como importantes. São eles:

- Empresa estabelecida no mercado (não ser recém fundada) (Ho, Dey \& Lockström, 2011);

- Empresa com reputação de Honestidade (Bhattacharya, Geraghty \& Young, 2010). Sendo que a reputação é considerada importante para a sua confiabilidade;

- A busca da melhoria contínua com relação à qualidade e a produtividade (Ho, Dey \& Lockström, 2011);

- Responsabilidade dos fornecedores pela qualidade da produção e dos módulos que eles fornecem (Rodrigues et al., 2012). Apesar disso, a terceirização de atividade não exime as empresas clientes das suas responsabilidades de manter o nível de qualidade atendendo todos os requisitos legais e os comprometidos com os consumidores (ABNT ISO/TS 16949:2010).

- "Ter operações flexíveis à demanda" (Carnevalli et al., 2013, p. 335) de modo a poder se ajustar num sistema de produção JIT (Just In Time);

- Preço competitivo (Rodrigues, 2005). Verifica-se que o preço continua sendo um fator importante a ser considerado na escolha dos fornecedores, mas não é mais o único fator;

- Transparência de longo prazo da Estratégia (Ho, Dey \& Lockström, 2011), o que permite verificar se as estratégias aplicadas em cada empresa não são contraditórias e se vão contribuir no atendimento dos objetivos das duas empresas;
Deste modo, a confiabilidade, qualidade, flexibilidade, transparência e preço do fornecedor, são qualidades exigidas importantes que os fornecedores devem ter ou desenvolver.

\subsection{Resultado da matriz da Qualidade em relação a tabela Características da Qualidade}

Com a realização da matriz da qualidade, após realizar as correlações entre as tabelas Qualidades Exigidas (QEs) e Características da Qualidade (CQs), foram identificadas as CQs mais importantes para os fornecedores aplicarem a estratégia modular. Estas estão relacionadas com: a qualificação dos funcionários, pelo número de funcionários que são treinados $\left(1^{a}\right.$ das Características da Qualidade mais importantes); número de funcionários experientes em cada área da empresa ( $2^{\text {a }}$ das CQs mais importantes) (Amin \& Razmi; 2009); anos de experiência na função ( $3^{\mathrm{a}}$ das CQs mais importantes) (Amin \& Razmi; 2009) e formação técnica dos funcionários "na área" (Sim/Não) (14 a das CQs mais importantes). Deste modo, a experiência, as competências técnicas e a capacitação dos funcionários são pontos importantes para desenvolver o know how da empresa. Isto porque esta experiência será necessária para desenvolver técnicas para definir os módulos pela função dos produtos ou pela estrutura do produto (Carnevalli et al., 2013) (21 ${ }^{\mathrm{a}}$ das CQs mais importantes) e ter Modularidade de Produto (Sim/Não)[Proponente] (22a das CQs mais importantes).

Como $4^{a}$ das CQs mais importantes apareceu a implementação da ABNT ISO/TS 16949:2010 (Sim/Não) a qual é pré-requisito básico, que qualquer fornecedor direto da montadora tem que atender, independente de ser fornecedor de módulo ou não. De qualquer maneira a norma TS ajuda a atender a $6^{\mathrm{a}}$ Característica da Qualidade: a implantação da Manutenção preventiva total 
(Zarei, Fakhrzad \& Paghaleh, 2011) que faz parte do requisito 7.5.1.4 da ABNT ISO/TS 16949:2010 e indiretamente a $5^{\text {a }}$ Característica da Qualidade: Entrega dos módulos e sistemas direto na linha de montagem da montadora (Prieto \& Miguel, 2011) (Sim/Não)". Isto porque se o fornecedor está entregando os módulos na linha de produção da montadora é porque o cliente está trabalhando com baixos estoques, podendo ser a produção de forma sincronizada com as entregas e qualquer atraso por quebra de máquina no fornecedor pode parar a linha de montagem da montadora. Por esta razão, não é de estranhar que a $7^{\mathrm{a}}, 8^{\mathrm{a}}, 10^{\mathrm{a}}, 13^{\mathrm{a}}$, $15^{\mathrm{a}}, 17^{\mathrm{a}}, 19^{\mathrm{a}}$ e $24^{\mathrm{a}}$ Característica da Qualidades (CQs) estejam relacionadas com aspectos da qualidade, as quais são:

- $7^{\mathrm{a}}$ CQs - Aplicação do 6 sigma (sim/não);

- $8^{a}$ CQs - Referência na satisfação dos clientes (Ho, Dey \& Lockström, 2011) (Sim/Não);

- $10^{\mathrm{a}} \mathrm{CQs}$ - Terceiriza a gestão da qualidade das peças dos módulos para os seus fornecedores (Prieto \& Miguel, 2011) (Sim/Não);

- $13^{\mathrm{a}}$ CQs - Ter programa TQM implementado (Ho et al., 2012; Zarei, Fakhrzad \& Paghaleh, 2011) (Sim/Não);

- $15^{\mathrm{a}} \mathrm{CQs}$ - Os fornecedores devem ter a norma ISO 9001:2008 (ABNT ISO/TS 16949:2010) (Sim/Não). Isto porque esta norma ajuda a empresa a padronizar e documentar os seus procedimentos, o que facilita a análise deste procedimento para manter e melhorar a qualidade;

- 16 $6^{\mathrm{C}}$ CQs - Avaliar o trade-off: qualidade Matéria-Prima/custos (\$);

- $17^{a}$ CQs - Conformidade com os requisitos do produto entregue (Sim/Não) (ABNT ISO/ TS 16949:2010, p.25);

- 19a CQs - Qualidade: Referências dos clientes atuais (Ho et al., 2012). (Sim/Não);

- $24^{\mathrm{a}} \mathrm{CQs}$ - Processo conforme com o projeto (Sim/Não).
Isto porque se o fornecedor não tiver altos níveis de qualidade, pode prejudicar os clientes devido a atrasos nas entregas dos módulos na linha de montagem ou até pior, a entrega de um módulo defeituoso. Outro aspecto interessante é que a metodologia do 6 sigma, as Normas ISO 9001, assim como a filosofia TQM exigem um investimento constante em melhoria e treinamento, sendo que treinamento foi a Característica da Qualidade mais importante identificada nesta análise.

A implantação de processos produtivos e de entregas programáticas JIT (Just In Time) (Zarei, Fakhrzad \& Paghaleh, 2011; ABNT ISO/TS 16949:2010) está em 11 ${ }^{a}$ CQs e ajuda atender diretamente o $5^{\text {a }}$ Característica da Qualidade: Entrega dos módulos e sistemas direto na linha de montagem da montadora (Prieto \& Miguel, 2011) (Sim/ Não)". Entretanto, implantar o JIT não é simples e exige ter recursos disponíveis para investir $\left(12^{\mathrm{a}}\right.$ CQs), em equipamento com capacidade adequada, sistemas de informações eficientes, programação de manutenção ( $6^{\mathrm{a}} \mathrm{CQs}$ ), ter nível de qualidade aceitável conforme as $7^{\mathrm{a}}, 10^{\mathrm{a}}, 13^{\mathrm{a}}, 15^{\mathrm{a}}, 17^{\mathrm{a}}$ e $24^{\mathrm{a}}$ CQs, fornecedores de peças e matérias primas confiáveis e investimentos constantes em treinamento conforme a $1^{\mathrm{a}} \mathrm{CQs}$.

A necessidade de entregar os módulos JIT ou JIS (Just-In-Sequence) de forma precisa incentiva estes fornecedores a criarem novas unidades de montagens, próximas das montadoras, seja dentro do condomínio industrial, consórcio modular ou simplesmente próximas dos parques industriais. Esta importância fica evidente quando se verifica na matriz da qualidade que as montadoras consideram a distância do fornecedor das suas plantas como um critério de seleção. Pois quanto mais próximo o fornecedor está da montadora, pode existir uma maior confiabilidade no cumprimento dos prazos de entregas ( $\mathrm{Ng}, 2008)$, sendo esta a $22^{a}$ CQs. Esta redução de distância também aju- 
da a atender a $18^{\mathrm{a}} \mathrm{CQs}$, avaliar o trade-off custo de estoque/transporte (\$) e a $21^{\mathrm{a}} \mathrm{CQs}$, verificar o uso de combinação de modais, com o objetivo de reduzir os custos.

A terceirização é outro ponto importante seja de fabricação de peças que fazem parte do módulo ou de gestão de qualidade destas peças, isto porque ao assumir a fabricação dos módulos, os fornecedores de primeiro nível começam a ter que trabalhar com vários componentes e peças que não fazem parte do seu know how. Sendo assim, estes poderão terceirizar parte de suas atividades para fornecedores de segundo nível, na fabricação destas peças (Prieto \& Miguel, 2011) (9a das CQs mais importantes). Ao terceirizar subsistemas e operações não chaves (Doran, 2002, 2003, 2004, 2005; Doran, Hill, Hwang, Ki-Soon, \& Jacob 2007) (23 ${ }^{a}$ das CQs mais importantes), a empresa poderá focar seus esforços no seu core business ou seja no desenvolvimento das suas competências centrais (Prieto \& Miguel, 2011) $\left(20^{\text {a }}\right.$ das CQs mais importantes) e na coordenação e integração destes componentes. Apesar disso, conforme a norma TS 16949:2010, a responsabilidade final pela qualidade continua sendo da empresa que terceirizou a atividade para seus fornecedores, sendo ela que vai responder diretamente para as montadoras caso ocorram falhas nos módulos fornecidos.

\subsection{Síntese dos principais resultados}

Será reapresentada uma sínteses dos principais resultados em relação às necessidades de investimentos na aplicação da modularidade, alterações de relacionamento entre as empresas devido ao uso da modularidade e critérios de responsabilidade pela qualidade dos módulos. Foram identificadas as seguintes necessidades para capacitar o fornecedor de $1^{\circ}$ nível na aplicação da modularidade (por meio da literatura):
1 Necessidades de investimentos para:

- Atingir níveis de qualidade que atendam todos os requisitos da montadora, pela implantação do 6 sigma ou TQM;

- Desenvolvimento das competências necessárias da força de trabalho da empresa para aplicar a modularidade;

- Desenvolver a eficiência na logística, permitindo um fornecimento JIT (Just In Time) e ou JIS (Just-In-Sequence);

- Ter recursos financeiros para investir na aplicação da modularidade;

2 Identificaram-se as alterações de relacionamento entre a empresa (fornecedor de $1^{\circ}$ nível) com seus fornecedores (fornecedor de $2^{\circ}$ nível) que são necessárias para a aplicação da modularidade:

- Verificou-se que o fornecedor de $1^{\circ}$ nível transferiu as operações não importantes para os seus fornecedores de $2^{\circ}$ nível (Doran, 2002, 2003, 2004, 2005; Doran et al., 2007) como a produção das peças (Prieto $\&$ Miguel, 2011). Deste modo, o abastecimento JIT (Just-In-Time) se torna um aspecto importante para permitir a montagem dos módulos no fornecedor de $1^{\circ}$ nível, de modo a possibilitar um abastecimento JIS (Just-InSequence) na planta da montadora;

3 Identificaram-se os critérios de responsabilidade pela qualidade dos módulos:

- Estes critérios estão definidos na norma TS: Segundo a norma (ABNT ISO/TS 16949:2010) a terceirização de atividade não exime a empresa das suas responsabilidades de manter o nível de qualidade atendendo todos os requisitos legais e os comprometidos com os clientes. Ou seja, a responsabilidade da qualidade dos módulos é do fornecedor 
dos módulos e não dos fornecedores de peças que formam os módulos.

- Segundo a norma (ABNT ISO/TS 16949:2010) na verificação dos produtos recebidos do fornecedor, deve-se: usar dados estatísticos; inspeção no recebimento com amostragem; auditoria de segunda ou terceira parte no fornecedor; e outras verificações definidas em contrato. Deste modo, cabe ao fornecedor de primeiro nível avaliar o nível de qualidade dos produtos dos fornecedores de segundo nível.

\section{Conclusões}

Com a construção da matriz da qualidade proposta nesta pesquisa verificou-se que os aspectos prioritários que os fornecedores devem desenvolver estão relacionados com os aspectos de gestão da qualidade e de RH. O objetivo é desenvolver o nível de qualidade e de know how necessários para os fornecedores poderem assumir atividades de projeto, fabricação, montagem e distribuição de módulos. No caso da qualidade, o fornecedor deve além de atender a ABNT ISO/ TS 16949:2010, buscar a melhoria contínua utilizando a metodologia do 6 sigma ou TQM. As necessidades de infraestrutura de equipamentos estão relacionados, principalmente, a aspectos de eficiência na logística e de trocas de informações que permitam um fornecimento JIS (Just-InSequence). Neste contexto, vale salientar que ter equipamentos de manufatura que permitam fabricar produtos com o nível de qualidade e flexibilidade exigidos, também é um aspecto fundamental para viabilizar o JIS. Uma questão fundamental é que qualquer empresa que queira ser fornecedora de módulos deve ter uma boa saúde financeira e capital disponível, para fazer a implantação dos recursos em qualidade, RH e logística. Assim, torna-se necessário criar uma estrutura adequada que permita atender as exigências da montadora em qualidade, treinamento, experiência na função e nível de qualificação, know how, logística, redução de custos e estabilidade financeira como pontos fundamentais para a empresa se tornar uma fornecedora de módulos. Somente após desenvolver estas competências, a empresa estará pronta para assumir como fornecedora de módulos numa relação de parceria estratégica com a montadora.

\section{Referências}

ABNT ISO/TS 16949:2010 (2009). Sistemas de Gestão da Qualidade - requisitos particulares para aplicação da ABNT NBR ISO 9001:2008 para organizações de produção automotiva e peças de reposição pertinentes. (2a ed.) Rio de Janeiro: ABNT.

Akao, Y. (1996) Introdução ao desdobramento da qualidade. Belo Horizonte: Fundação Christiano Ottoni.

Amin, S.H., \& Razmi, J. (2009). An integrated fuzzy model for supplier management: A case study of ISP selection and evaluation. Expert Systems with Applications, 36(4), 8639-8648. doi: http://doi. org/10.1016/j.eswa.2008.10.012

Arnheiter, E.D., \& Harren, H. (2005). A typology to unleash the potential of modularity. Journal of Manufacturing Technology Management, 16(7), 699711. doi: 10.1108/17410380510619923

Asan, U., Polat S., \& Serdar S. (2004). An integrated method for designing modular products. Journal of Manufacturing Technology management, 15 (1), 29-49. doi: http://doi.org/10.1108/09576060410512257

Baldwin, C.Y., \& Clark K.B. (2000). Design rules. Massachusetts: MIT Press.

Bhattacharya, A., Geraghty, J., \& Young, P. (2010). Supplier selection paradigm: An integrated hierarchical QFD methodology under multiple-criteria environment. Applied Soft Computing, 10 (4), 1013-1027. doi: http:// doi.org/10.1016/j.asoc.2010.05.025

Borghi, R.A.Z. (2011). Economia financeira e economia produtiva: o padrão de financiamento da indústria automobilística. Dissertação de mestrado, Universidade de Campinas, Campinas, SP, Brasil.

Brad S. (2008). Vectors of innovation to support quality initiatives in the framework of ISO 9001:2000. International Journal of Quality \& Reliability Management, 25 (7), 674-693. doi: http://doi. org/10.1108/02656710810890872 
Carnevalli, J.A., Miguel, P.A.C., \& Salerno, M. S. (2013) Aplicação da modularidade na indústria automobilística: análise a partir de um levantamento tipo survey. Produção, 23 (2), 329-344. doi: http://doi.org/10.1590/ S0103-65132012005000040

Carnevalli, J.A., Miguel, P.A.C., \& Calarge, F.A. (2010). Axiomatic design application for minimising the difficulties of QFD usage. International Journal of Production Economics. 125, 1 - 12. doi: http://doi. org/10.1016/j.ijpe.2010.01.002 .

Celik, M., Cebi, S., Kahraman, C., \& Er, D.I. (2009). An integrated fuzzy QFD model proposal on routing of shipping investment decisions in crude oil tanker market. Expert Systems with Applications, 36(3), 6227-6235. doi: http://doi.org/10.1016/j.eswa.2008.07.031

Cheng, L.C., \& Melo Filho, L.D.R. (2007). QFD: Desdobramento da Função Qualidade na gestão de desenvolvimento de produtos. São Paulo: Editora Blucher.

Rodrigues, E. de O. (2005). Metodologia para formação de parceria em cadeia de suprimentos: enfoque por similaridade entre atividades. (tese de doutorado, Universidade Estadual de Campinas). Retrieved from http://repositorio.unicamp.br/jspui/bitstream/ REPOSIP/263451/1/Rodrigues,\%20Elder\%20de\%20 Oliveira.pdf

Collins, R., Bechler, K., \& Pires, S. R.I. (1997). Outsourcing in the automotive industry: from JIT to Modular Consortia. European Management Journal, 15(5), 498-508. doi: https://doi.org/10.1016/S02632373(97)00030-3

Doran, D. (2003). Supply Chain Implications of Modularization. International Journal of Operations and Production Management. 23(3), 316-326. doi: 10.1108/01443570310462785

Doran, D. (2002). Manufacturing for synchronous supply: a case study of Ikeda Hoover Ltd. Integrated Manufacturing Systems, 13, (1), 18-24. DOI: http://doi. org/10.1108/09576060210411477

Doran, D. (2004). Rethinking the supply chain: na automotive perspective. Supply Chain Management: An International Journal, 9(1), 102-109. doi: http://doi. org/10.1108/13598540410517610

Doran, D. (2005). Supplying on a modular basis: an examination of strategic issues. International Journal of Physical Distribution \& Logistics Management, 35 (9), 654-663. doi: http://doi. org/10.1108/09600030510632023

Doran, D., Hill, A., Hwang, Ki-Soon, \& Jacob, G. (2007). Supply chain modularisation: Cases from the French automobile industry. International Journal of Production Economics, 106, (1), 2-11. doi: http://doi. org/10.1016/j.ijpe.2006.04.006
Elphick, P. (2006). Liberty: the ships that won the war. London: Chatham Publishing.

Frigant, V., \& Lung, Y. (2002) Geografical proximity and supplying relationships in modular production. International Journal of Urban and Regional Research, 26(4), 742-755. doi: http://doi.org/10.1111/14682427.00415

Gadde, L.E., \& Jellbo O. (2002). System sourcing - opportunities and problems. European Journal of Purchasing \& Supply Management, 8(1), 43-51. Retrieved from http://www.sciencedirect.com/science/ journal/09697012/8/1

Gonzalez, M. E., Quesada, G., Gourdin, K., \& Hartley, M. (2008). Designing a supply chain management academic curriculum using QFD and benchmarking. Quality Assurance in Education, 16 (1), 36-60. doi: http://doi.org/10.1108/09684880810848404

Grauer, M. (2007). Globally distribute manufacturing networks: Interdependencies Between Product Design And Choice Of Production Site. Paper presented at the meeting of Proceedings of The World Congress on Engineering and Computer Science, San Francisco, USA. Retrived from http://www.iaeng.org/publication/ wcecs2007/wcecs2007_pp995-998.pdf

Ho, W., He, T., Lee, C.K.M., \& Emrouzneja, D.A. (2012). Strategic logistics outsourcing: An integrated QFD and fuzzy AHP approach. Expert Systems with Applications, 39, (12), 10841-10850. doi: http://doi. otg/10.1016/j.eswa.2012.03.009

Ho,W., Dey, P.K., \& Lockström, M. (2011). Strategic sourcing: a combined QFD and AHP approach in manufacturing. Supply Chain Management: An International Journal, 16 (6), 446-461. doi: http://doi. org/10.1016/j.ijpe.2015.03.028

Ho, W., Xu, X., \& Dey, P.K. (2010). Multi-Criteria Decision Making approaches for supplier evaluation and selection: A literature review. European Journal of Operational Research, 202(1), 16-24. doi: http://doi. org/10.1016/j.ejor.2009.05.009

Hoek, R.I.V., \& Weken, H.A.M. (1998). The impact of modular production on the dynamics of supply chains. The International Journal of Logistics Management, 9(2), 35-50. doi: 10.1108/09574099810805825

Jia, G.Z., \& Bai, M., (2011). An approach for manufacturing strategy development based on fuzzyQFD. Computers \& Industrial Engineering, 60 (3), $445-$ 454. doi: http://doi.org/10.1016/j.cie.2010.07.003

Katta, G.D.P., Kambagowni, V.S., \& Kandukuri N.R. (2012). Aligning The Competitive Strategy with Supply Chain Strategy Through QFD. Journal of Advances in Management Research, 9(2), 189-198. doi: http://doi. org/10.1108/09727981211271931 
Killen, C.P., Walker, M., \& Hunt, R.A. (2005). Strategic planning using QFD. International Journal Of Quality \& Reliability Management, 22 (1), 17-29. doi: http://doi. org10.1108/02656710510572968

Lam, J. S. L., \& Lai, K. L. (2015). Developing environmental sustainability by ANP-QFD approach: the case of shipping operations. Journal of Cleaner Production, 105, 275-284. doi: http://doi.org/10.1016/j. jclepro.2014.09.070

Larsson, A. (2002). The development and regional significance of the automotive industry: supplier parks in western Europe. International Journal of Urban and Regional Research, 26(4), 767-784. doi: 10.1111/14682427.00417

Marconi, M. D. A., \& Lakatos, E. M. (2002). Técnicas de pesquisa: planejamento e execução de pesquisas, amostragens e técnicas de pesquisas, elaboração, análise e interpretação de dados. (5a ed.) São Paulo: Atlas.

Miguel, F. L. P. (2009). As estratégias de compras das multinacionais automobilísticas: um estudo de caso da PSA Peugeot Citroën no Rio de Janeiro. (Dissertação de doutorado, Universidade Federal do Rio de Janeiro). Retrieved from http://www. ie.ufrj.br/images/pesquisa/publicacoes/teses/2009/ AS\%20ESTRAT\%C3\%89GIAS\%20DE $\% 20$ COMPRAS\%20DAS\%20MULTINACIONAIS $\% 20$ AUTOMOBIL\%C3\%8DSTICA.pdf

Morris, D., \& Donnelly, T. (2006). Are there market limits to modularisation? International Journal of Automotive Technology and Management, 6(3), 262275. doi: http://doi.org/10.1504/IJATM.2006.012120

$\mathrm{Ng}$, W. L. (2008). An efficient and simple model for multiple criteria supplier selection problem. European Journal of Operational Research, 186, 1059-1067. doi: http://doi.org/10.1016/j.ejor.2007.01.018

Ni, M., \& Deng, S. (2007). Extended QFD and datamining-based methods for supplier selection in mass customization. International Journal of Computer Integrated Manufacturing, 20(2-3), 208-291. doi: http:// doi.org/10.1080/09511920601150651

Pandremenos, J., Paralikas, J., Salonitis, K., \& Chryssolouriset, G. (2009). Modularity concepts for the automotive industry: a critical review. CIRP Journal of Manufacturing Science and Technology, 1(3), 148-152. doi: 10.1016/j.cirpj.2008.09.012

Park, J., Shin, D., Insun, P., \& Hyemi, H. (2008). A product platform concept development method. Journal of Engineering Design, 19(6), 515-532. doi: http://doir. org/10.1080/09544820802043583

Prieto, E., \& Miguel, P.A.C. (2011). Adoção da estratégia modular por empresas do setor automotivo e as implicações relativas à transferência de atividades no desenvolvimento de produto: um estudo de casos múltiplos. Gestão \& Produção, 18, (2), 425-442. doi: http://doi.org/10.1590/S0104-530X2011000200015
Pires, S.R.I. (1998). Managerial implications of the modular consortium model in a Brazilian automotive plant. International Journal of Operations \& Production Management, 18(3), 221-232. doi: http://doi. org/10.1108/01443579810368290

Pires, S.R.I. (2002). New Productive Systems in the Automotive Industry: the current situation of three innovative plants in Brazil. International Journal of Automotive Technology and Managemen, 2, (1), 46-62. doi: 10.1504/IJATM.2002.000056

Pires, S.R. (2009). Gestão da cadeia de suprimentos (supply chain management): conceitos, estratégias, práticas e casos (2nd ed.) São Paulo: Atlas.

Pires, S. R. I., \& Sacomano, M., Neto. (2010). Características estruturais, relacionais e gerenciais na cadeia de suprimentos de um condomínio industrial na indústria automobilística. Produção, 20(2), 172-185. doi: 10.1590/S0103-65132010005000032

Pur, M.M; Tabriz, A.A. (2012). SWOT Analysis using of Modified Fuzzy QFD - A Case Study for Strategy Formulation in Petrokaran Film Factory. Procedia Social and Behavioral Sciences, 41, 322-333. doi: http:// dx.doi.org/10.1016/j.sbspro.2012.04.037

Rabelo, M. M. (2011). Elaboração e aplicação de método para escolba de fontes de fornecimento para Indústria de autopeças. (Dissertação de Mestrado Profissional, Universidade Estadual de Campinas). Retrieved from http://www.bibliotecadigital.unicamp.br/ document $/$ ?code $=000876056$

Rodrigues, E. O. (2005). Metodologia para formação de parceria em cadeia de suprimentos: enfoque por similaridade entre atividades. (Dissertação de doutorado, Universidade Estadual de Campinas). Retrieved from http://www.bibliotecadigital.unicamp.br/ document/?code $=\mathrm{vtl}$ s000374466

Rodrigues, E.A., Carnevalli, J.A., \& Miguel, P.A.C. (2012). Uma investigação sobre a relação entre o projeto do produto e produção em uma montadora automotiva e fornecedores de motores que adotam a modularidade. Produção, 22 (3), 367-379. doi: 10.1590/S010365132012005000019

Salari, M., \& Bhuiyan, N. (2016). A new model of sustainable product development process for making trade-offs. The International Journal of Advanced Manufacturing Technology. 86, (1-4), p.11. doi: http:// doi.org/10.1007/s00170-016-9349-y

Salerno, M. S., Marx, R., Zilbovicius, M., \& Dias, A.V.C. (2009). The importance of locally commanded design for the consolidation of local supply chain: the concept of design headquarters. International Journal of Automotive Technology and Management, 16(4), 361376. : doi: 10.1504/IJMTM.2009.023753 
Segismundo. A., \& Cauchick, P.A.M. (2014). Análise da descentralização no desenvolvimento de novos produtos: uma investigação em uma empresa do setor de veículos comerciais. Produção, 24(1), 118-131. doi: 10.1590/ S0103-65132013005000014

Sorroor, J. Tarokh, M. J., \& Abedzadeh, M. (2012). Automated bid ranking for decentralized coordination of construction logistics. Automation in Construction, 24, 111-119, 2012. doi : http://doi.org/10.1016/j. autcon.2011.11.013

Tontini, G. (2003). Deployment of customer needs in the qfd using a modified Kano model. Journal of Academy of Business and Economics, 2(1), 103-113.

Tontini, G. (2007). Integrating the Kano model and QFD for designing new products. Total Quality Management \& Business Excellence, 18(6), 599-612. Doi: http://doi. org/10.1080/14783360701349351

Veloso, F., \& Fixson, S. (2001). Make-Buy decisions in the auto industry: new perspectives on the role of the supplier as an innovator. Technological Forecasting and Social Change, 67, (2-3), 239-257. https://doi. org/10.1016/S0040-1625(00)00092-5
Zarei, M., Fakhrzad, M.B., \& Paghaleh, M.J. (2011). Food supply chain leanness using a developed QFD model. Journal of Food Engineering, 101 (1), 25-33. doi: http://doi.org/10.1016/j.jfoodeng.2010.07.026

Zohbabi, A., \& Manteghi, N. (2011). A proposed model for strategia planning in educational organizations. Procedia-Social And Behavioral Sciences, 28, 205-210. doi: http://doi.org/10.1016/j.sbspro.2011.11.039

Yücel, A., \& Güneri, A.F. (2011). A weighted additive fuzzy programming approach for multi-criteria supplier selection. Expert Systems with Applications, 38 (5), 6281-6282. doi: http://doi.org/10.1016/j. eswa.2010.11.086

Wang, C.H, \& Chen, J.N. (2012) Using quality function deployment for collaborative product design and optimal selection of module mix. Computers \& Industrial Engineering, 63 (4), 1030-1037. doi: http://doi. org/10.1016/j.cie.2012.06.014

Recebido em 18 mai. 2017 / aprovado em 14 mar. 2018

Para referenciar este texto

Carnevalli, J. A., Sousa, J. E. R., Benedicto, S. C., Medeiros, C. G. A., \& Georges, M. R. R. Uso do QFD para definir requisitos necessários para a aplicação da estratégia da modularidade nos fornecedores de primeiro nível do setor automotivo. Exacta, São Paulo, v. 16, n. 4, p. 149-163. out./dez. 2018. Disponível em: <https://doi.org/10.5585/ExactaEP.v16n4.7426> 
H..... . . . 